\title{
Automated Analysis of the Distributions and Geometries of Blood Vessels on Retinal Fundus Images
}

\author{
Yuji Hatanaka*a, Takeshi Harab ${ }^{\mathrm{b}}$, Hiroshi Fujita ${ }^{\mathrm{b}}$, Masaru Aoyama ${ }^{\mathrm{c}}$, \\ Hideya Uchida ${ }^{\mathrm{d}}$, Testuya Yamamoto ${ }^{\mathrm{c}}$ \\ ${ }^{a}$ Dept. of Electronic Control Engineering, Gifu National College of Technology, \\ 2236-2 Kamimakuwa, Motosu, Gifu, Japan 501-0495; \\ ${ }^{b}$ Dept. of Intelligent Image Information, Division of Regeneration and Advanced Med. Science, \\ Graduate School of Medicine, Gifu University, 1-1 Yanagido, Gifu, Japan 501-1193; \\ ${ }^{c}$ Dept. of Ophthalmology, Gifu University, School of Medicine, 40 Tsukasa-machi, Gifu, Japan \\ 500-8705; \\ ${ }^{\mathrm{d}}$ Uchida Eye Clinic, 4-18 Tetsumei Street, Gifu, Japan 500-8879
}

\begin{abstract}
We have developed a computer-aided diagnosis system to detect the abnormalities on retinal fundus images. In Japan, ophthalmologists usually detect hypertensive changes by identifying narrowing arteriolae with a focus on an irregularity. The purpose of this study is to develop an automated method for detecting narrowing arteriolae with a focus on an irregularity on retinal images. The blood vessel candidates were detected by the density analysis method. In blood vessel tracking, a local detection function was used to go along the centerline of the blood vessel. A direction comparison function using three vectors was designed to provide an optimal estimation of the next possible location of a blood vessel. After the connectivity of vessel segments was adjusted based on the recognized intersections, the true treelike structure of the retinal blood vessels was established. The abnormal blood vessels were finally detected by measuring their diameters. The comparison between the results obtained using our system and the diagnostic results of physicians showed that our proposed method automatically detected an irregularity in diameter in $75 \%$ of all 24 narrowing arteries with a focus on an irregularity on 70 retinal fundus images. Approximately 2.88 normal vessel segments per image were determined to be abnormal, a number which must be reduced at the next stage. The automated detection of narrowing arteriolae with a focus on an irregularity could help ophthalmologists in diagnosing ocular diseases.
\end{abstract}

Keywords: Retinal fundus image, blood vessel, vascular tree tracking, geometric analysis, computer-aided diagnosis

\section{INTRODUCTION}

The number of patients with adult diseases including cardiac and cerebrovascular diseases is currently on the increase in our country. To prevent or detect these diseases at an early stage, ophthalmologists rely on retinal fundus images or direct ophthalmoscopy during total health examinations or mass screenings. In recent times, ophthalmoscopy has been the third most frequently used method of examination in health examinations after sphygmomanometry and electrocardiography. Generally, in our country, ophthalmologists visually observe retinal fundus images in diagnosing hypertension. Ophthalmoscopy is expected to be the most frequently used one in the future. The frequent use of this technique, however, may impose a heavy burden on ophthalmologists. Therefore, many research groups have made efforts in developing their own computer-aided diagnosis (CAD) systems based on retinal fundus images [1-7].

In our country, ophthalmologists usually diagnose hypertensive retinopathy and arteriolosclerosis according to the classification of Scheie. This classification determines the severity (I to IV) of atherosclerotic and hypertensive changes. In most cases, ophthalmologists diagnose these diseases by observing the condition of vessels. This does not allow for quantitative determination. Therefore, the objective of this study is to develop an automatic analysis method for observing blood vessel distributions and geometries on retinal fundus images.

Recently, many studies on the CAD system for fundus images have been reported [1-7]. Tolias et al. presented a method for detecting blood vessels [1]. Their fuzzy algorithm for vessel tracking was applied to the detection of ocular 
fundus vessels. Their main tool identifies vessel and non-vessel regions on a vessel profile if the fuzzy C-means clustering algorithm is enabled with properly pre-processed data entered. Zana et al. presented an algorithm for the temporal and/or multimodal registration of fundus images based on point correspondence [2]. This technique used the Hough Transform to analyze the furcations. Chaudhuri et al. presented a method for detecting blood vessels [3]. They developed 12 different templates intended for use in searching for vessel segments in all possible directions. Can et al. presented an algorithm for vessel tracking and feature extraction from fundus images. Their algorithm tracked vessels using a set of two-dimensional correlation kernels that act as low-pass differentiators perpendicular to the direction in which the vessels run and to the low-pass averaging filters along the vessels. Sakuma et al. presented a method for correcting colors and detecting any changes based on the values for differences in sequential ocular retinal images [5]. The method we propose detects vessel furcations [6]. Our algorithm extracts vessels through a double-ring filter. This filter enables us to extract the vessels with a focus on an irregularity without being affected by narrowing vessels. We also propose an automated method for detecting eyeground hemorrhage and exudates on fundus images [7]. The results of the study on the method for detecting abnormal blood vessels having narrowing arteriolae with a focus on an irregularity have not been reported. To automate the "classification of Scheie" process, this study was designed to develop an automated method for detecting narrowing arteriolae on fundus images with a focus on an irregularity as shown in Fig. 1.

\section{METHODS}

\subsection{Overall scheme}

Our detection scheme including the new technique consisted of an 7-step procedure is followed: (1) Image digitization (1,600 dots per inch (dpi) and 24-bit color resolution), (2) pre-processing, (3) extraction of optic disks, (4) extraction of vessels, (5) tracking of vascular trees, (6) measurement of vessel diameters, and (7) detection of abnormal vessels.

\subsection{Image digitization, pre-processing of fundus images, and detection of optic disks}

The fundus images were captured on $35 \mathrm{~mm}$ positive film and digitized by the EPSON ES2000, a flatbed-type scanner with a transmissive unit. The digitized 24-bit color images are represented at a resolution of 1,600 $\times 1,600$ pixels in the bitmap format.

The scale of the matrix was first reduced to $800 \times 800$ pixels by taking detailed subsamples of the original image data for better processing efficiency.

Next, the color images were converted to grayscale using only the green component, thus achieving a clearer contrast compared with the original ones represented using all color components (Fig. 2 (a)).

Following this, as the optic disk was highlighted with a greater brightness than those of other tissues, it was extracted by the p-tile technique. The optic disk was removed from the grayscale image.

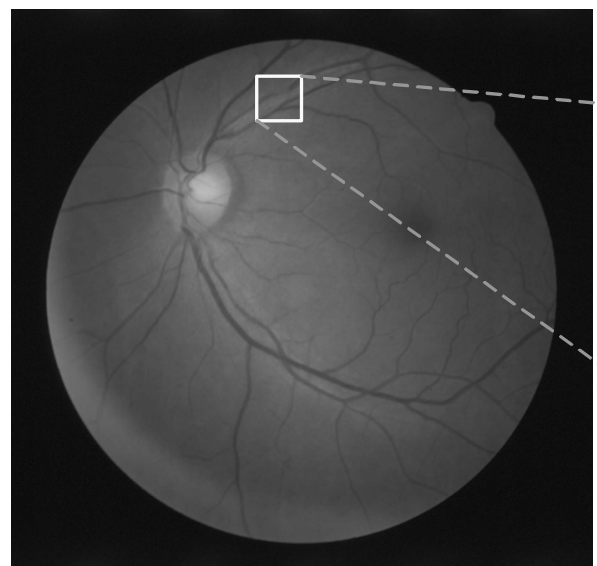

(a)

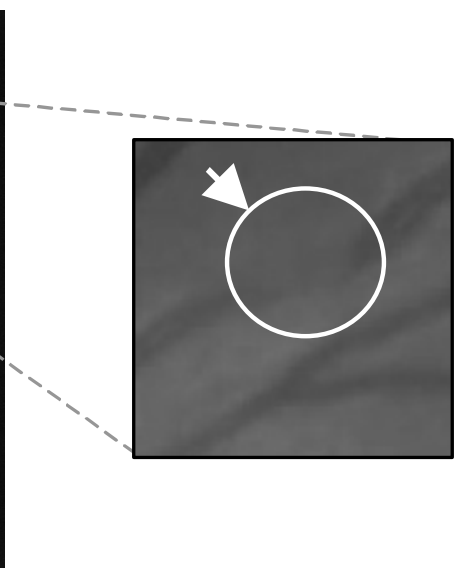

(b)

Fig. 1 An example of a narrowing arteriolar with a focus on irregularities. (a) An original fundus image including a narrowing arteriolar with a focus on an irregularity. (b) The enlarged image of a rectangle region shown in (a). 


\subsection{Extraction of vessels}

The blood vessel candidates were extracted by the threshold technique using a double-ring filter as shown in Fig. 3 [6]. Regions $\mathrm{A}$ and $\mathrm{B}$ had diameter of 3 pixels and 7 pixels, respectively. The feature value was calculated from the difference in the average value of pixels between regions $\mathrm{A}$ and $\mathrm{B}$. The threshold value for the feature value was experimentally set to 5. In Fig. 2 (b), an example of how to extract vessels is shown.

Next, many false candidates were eliminated to enable the extraction of only the main vessels. The false vessel candidates which were not connected with the optical disc and were isolated were eliminated. In Fig. 2 (c), an example of the processed image is shown.

\subsection{Vascular tree tracking}

In this step, the main vessels to be observed for diagnosis were detected by tracking the vessel candidates extending from the optic disk.

First, the centerlines of the vessel were extracted by the thinning algorithm. Next, the extracted vessels were tracked along their centerlines from the optic disk. The retinal vessels have several branches and furcations, which are determined by the number of neighboring pixels. The point with two neighboring pixels was defined as one of the elements of a straight line as shown in Fig. 4 (a); one with three neighboring pixels was defined as a bifurcation as

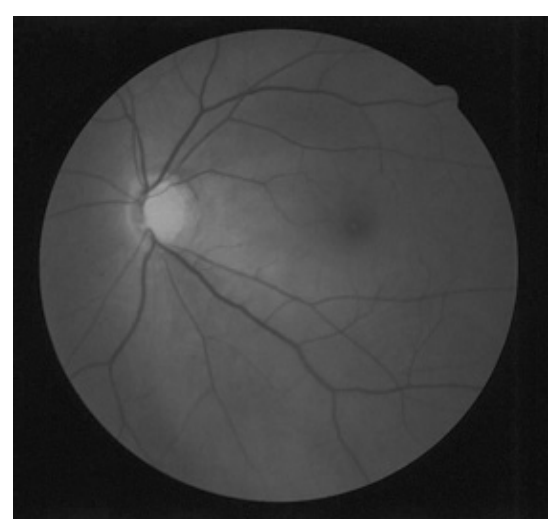

(a)

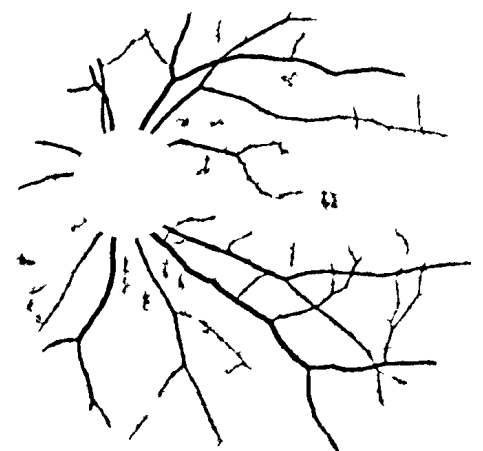

(b)

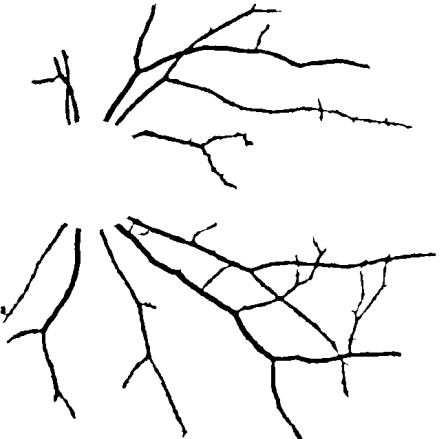

(c)

Fig. 2 These images have been pre-processed by this technique. (a) A gray scale (green-component) image. (b) Black lines indicate vessels extracted through a double-ring. (c) Extracted vessel candidates leading to false analysis were removed. On this image, only the main vessels and their veins and arteries are extracted.

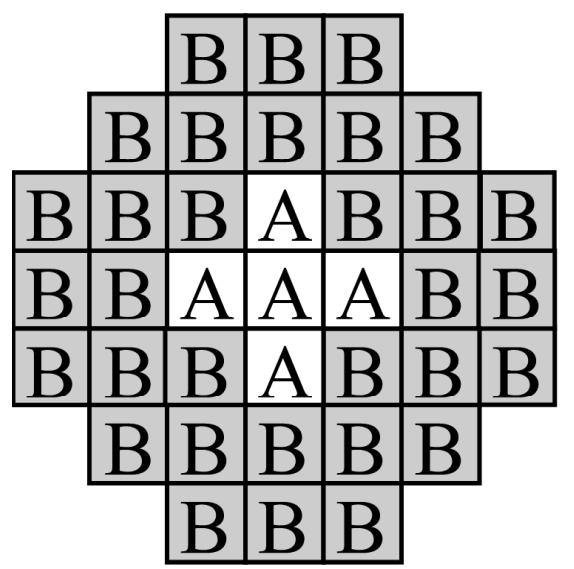

Fig. 3 A double-ring filter for extracting vessels. 
shown in Fig. 4 (c); and one with four neighboring pixels was defined as a furcation as shown in Fig. 4 (b).

In the next step, the direction of a vector was determined by the angle made by a bifurcation point and a furcation. An example of vector tracking is shown in Fig. 5. By our method, it was possible to track the vector if the internal angle $(\theta)$ of each branch was over 90 degrees. Table 1 shows the rule for vector tracking according to our method as shown in Fig. 5. For example, as the internal angle defined between branches A and C was approximately 180 degrees, and in our method, the vessel was tracked from branches A to $\mathrm{C}$. As the angles between branches $\mathrm{C}$ and $\mathrm{F}$ and between branches $\mathrm{A}$ and $\mathrm{F}$ were both over 90 degree, the vessel was tracked from branches $\mathrm{A}$ to F. However, as the angle between branches $A$ and $G$ was under 90 degrees, the vessel was not tracked from branches A to G.

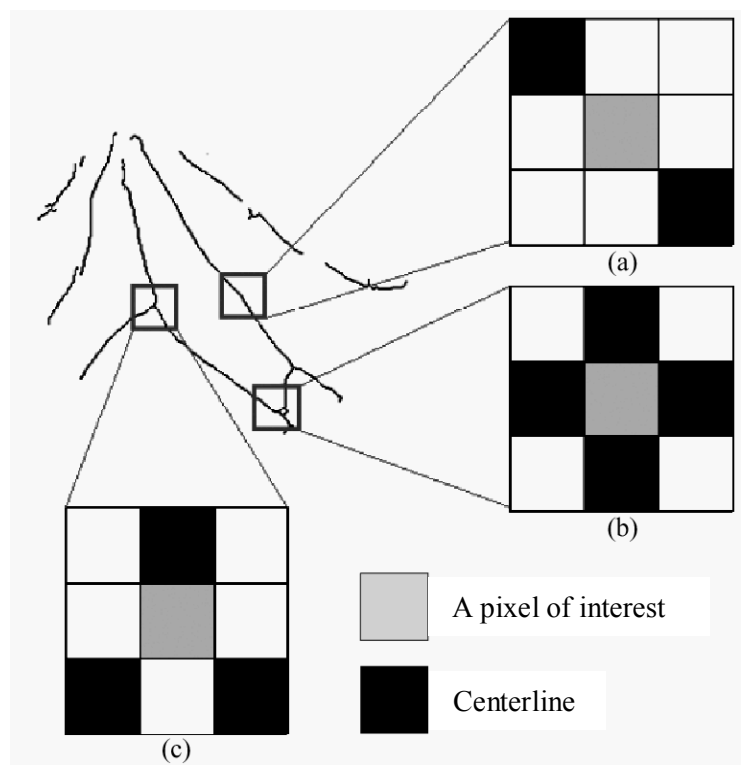

Fig. 4 Determination of furcations and branches. (a) Straight segment. (b) Furcation point. (c) Bifurcation.

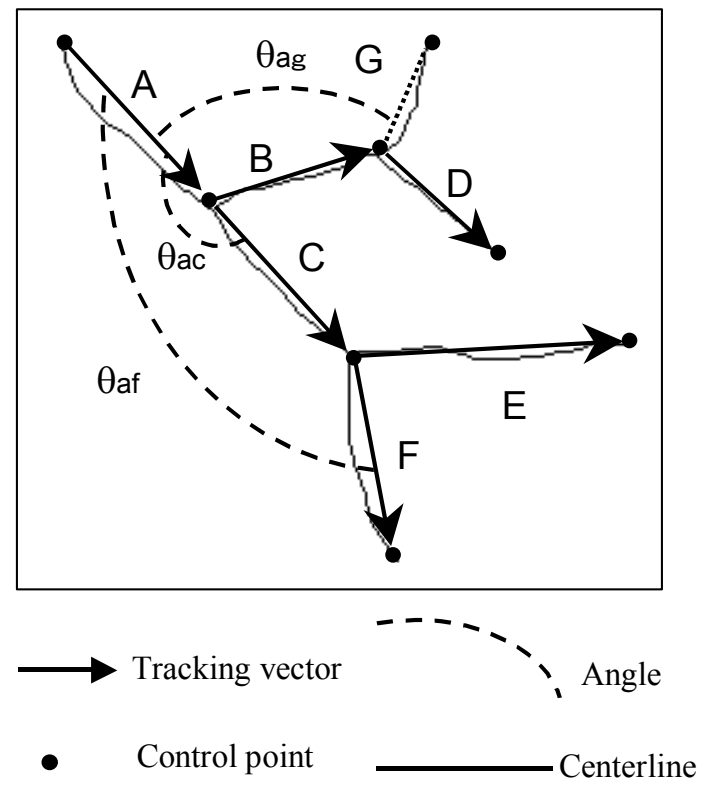

Table 1 Decision regarding the points for tracking vessels

\begin{tabular}{c|c|c|c}
\hline Successor & Requirement 1 & Requirement 2 & Action \\
\hline $\mathrm{E}$ & $\theta \mathrm{ce}>90^{\circ}$ & $\theta \mathrm{ae}>90^{\circ}$ & $\mathrm{O}$ \\
$\mathrm{F}$ & $\theta \mathrm{cf}>90^{\circ}$ & $\theta \mathrm{af}>90^{\circ}$ & $\mathrm{O}$ \\
$\mathrm{G}$ & $\theta \mathrm{bg}>90^{\circ}$ & $\theta \mathrm{ag}<90^{\circ}$ & $\times$ \\
$\mathrm{D}$ & $\theta \mathrm{bd}>90^{\circ}$ & $\theta \mathrm{ad}>90^{\circ}$ & $\mathrm{O}$ \\
\hline
\end{tabular}

Fig. 5 The method of vascular tree tracking. 


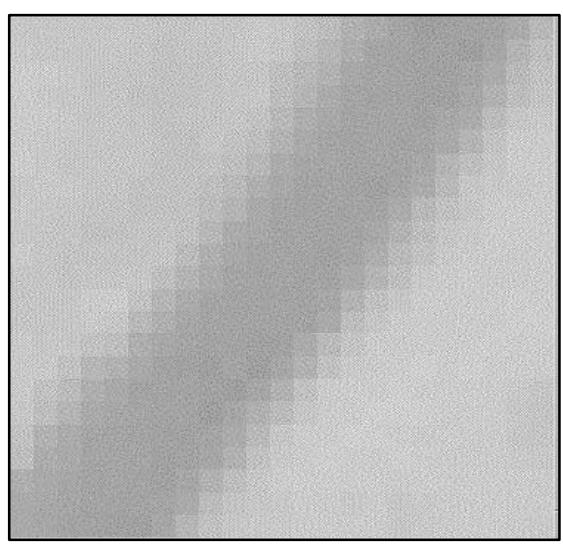

(a)

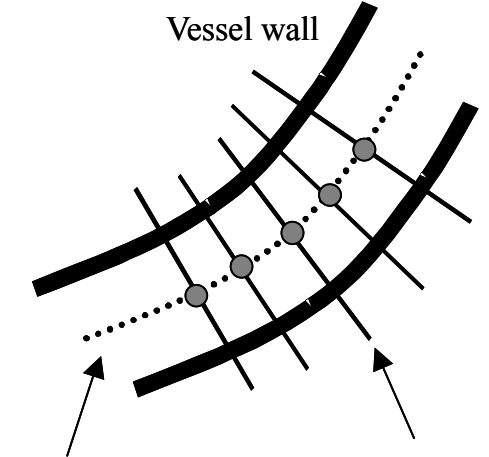

Centerline
Approximated line

(b)

Fig.6 (a) The retinal region of interest (ROI) on a gray image. (b) Measuring vessel width. The bold black lines indicate vessel walls, the black dashed line indicates the centerline of the vessel, and the black lines are perpendicular lines.

\subsection{Measurement of vessel diameters and detection of abnormal vessels}

Fig. 6 and Fig. 7 show the measurements of vessel diameters. First, the straight line was approximated using two neighboring pixels on its centerline. In the next step, a vertical line was determined using the approximated line of the centerline (Fig. 6 (b)). In the third step, the vessel walls were determined using the zero-crossing method [8]. It is well known that the zero-crossing method is effective in detecting edges. In Fig. 7, the vessel profile and Laplacian of the vessels are shown. The vessel wall indicates the point at which the Laplacian value was 0 (zero). Finally, the vessel diameter was measured based on the distance between the vessel walls on the approximated line (as shown in Fig. 7).

\subsection{Detection of abnormal vessels}

In this final step, the average vessel diameter of each branch was calculated. The vessels were determined to be abnormal if their vessel diameter in any section was narrower than $2 / 3$ rd the average diameter. An example of vessel diameter measurement is shown in Fig. 8. If the vessel was narrower than $2 / 3 \mathrm{rd}$ the average diameter in a certain segment, the vessel including the narrow segment was defined as abnormal. The example in Fig. 9 shows the result of abnormal vessel detection.

\section{RESULTS AND DISCUSSION}

To evaluate our method for detecting abnormal blood vessels, we examined 70 retinal fundus images, among which abnormal blood vessels were detected on 24 images. In all 24 abnormal cases, narrowing arteries with focal irregularities were observed, and no abnormal cases were detected in the remaining 46 images.

First, we evaluated our method for detecting vessels. An example of the result of vessel detection is shown in Fig. 10 (a). Most main vessels could be detected using our method; however, many false vessel candidates were also included. Next, we evaluated our method for tracking vessels. An example of the result of vessel tracking is shown in Fig. 10 (b). Each vessel could be outstandingly recognized without any confusion with other vessels, because the number of false vessel candidates was less than that shown in Fig. 10 (a). On the contrary, some false centerlines of blood vessels were miss-detected by the thinning algorithm. Accordingly, an efficient alternative thinning technique should be identified or developed to obtain the accurate centerlines of the blood vessels. Such findings suggest that our method may successfully work on the retinal CAD system if the known problems in our method are resolved.

Next, by comparing with the obtained results with the diagnostic results of physicians, it was demonstrated that this computerized method automatically detected any irregularity, in $75 \%$ of all 24 abnormal main blood vessels, and the remaining $25 \%$ ( 6 abnormal vessels) were not detected. Approximately 2.88 normal vessel segments per image were determined to be abnormal. We used a Pentium III-mounted (700 MHz) computer with 1,024 MB memory operating in the Solaris8 environment. Processing each image required approximately 70 to 90 seconds. Our retinal CAD system may work even more efficiently using a state-of-the-art computer. The recent results of the examination suggested that 
abnormal blood vessels were more likely to be detected by using the automated method abnormal blood vessels were more likely to be detected by using the automated method.

The analysis of blood vessel on retinal fundus images is significant in detecting abnormalities in vessels. Our scheme focused on the recognition of the whole blood vessel structure instead of partial vessel segments. Such a scheme provided the basis for an accurate quantitative analysis of the geometric features of blood vessels.

However, we also encountered some problems in the course of testing the scheme. Some vessel segments were broken after the density analysis. This might adversely affect vascular tree tracking. In addition, some vessel paths ran

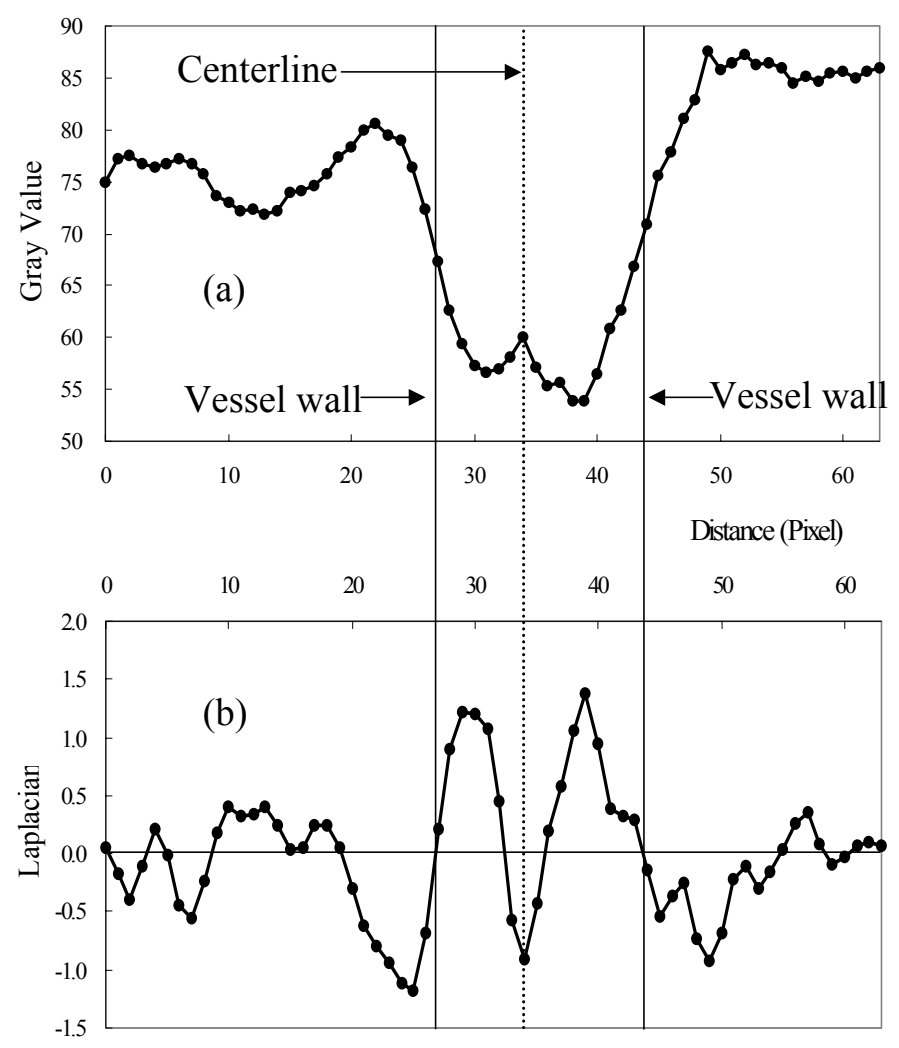

Fig. 7 The calculation of vessel diameters. (a) Vessel profile. (b) Laplacian of vessel profile. Vessel walls are determined using the zero-crossing method.

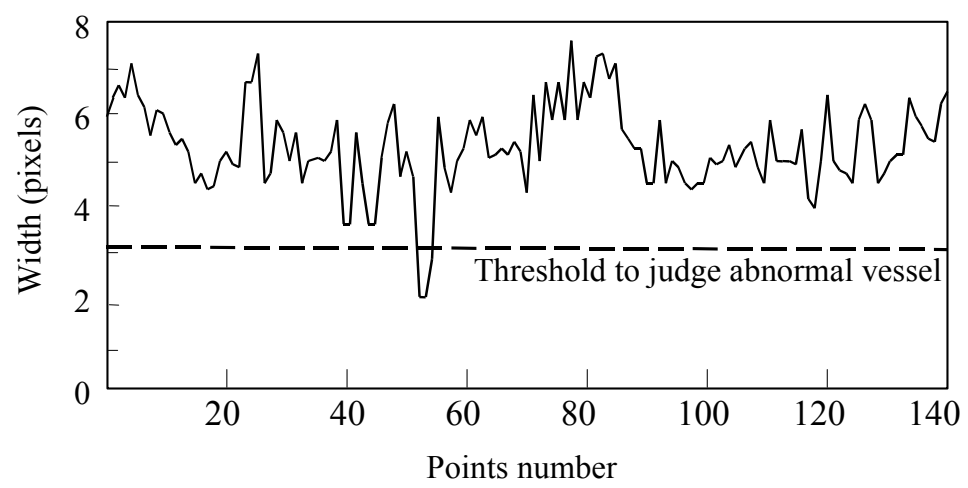

Fig. 8 Width of vessel segment. 


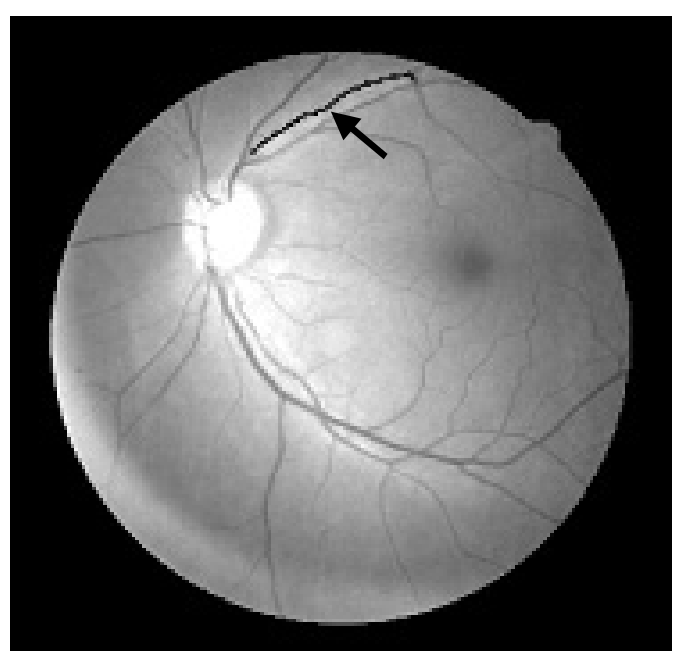

Fig. 9 The detected narrowing arteriola with a focus on an irregular segment indicated by a black arrow on a retinal fundus image.

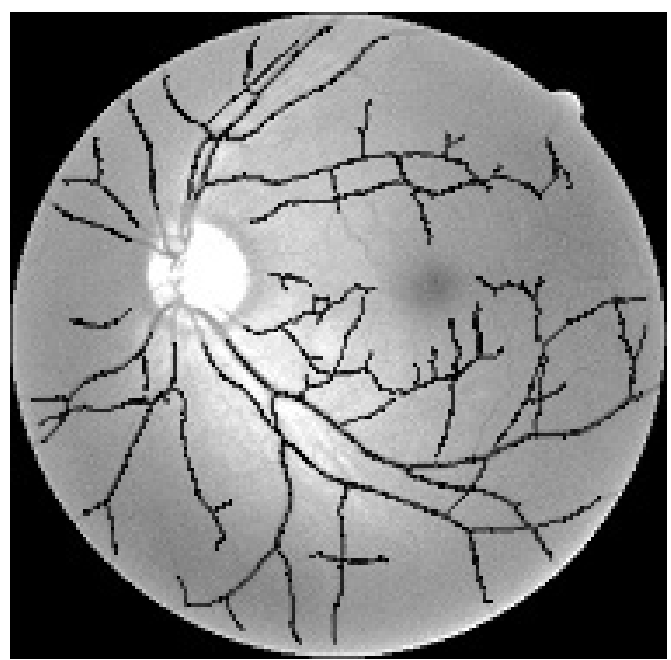

(a)

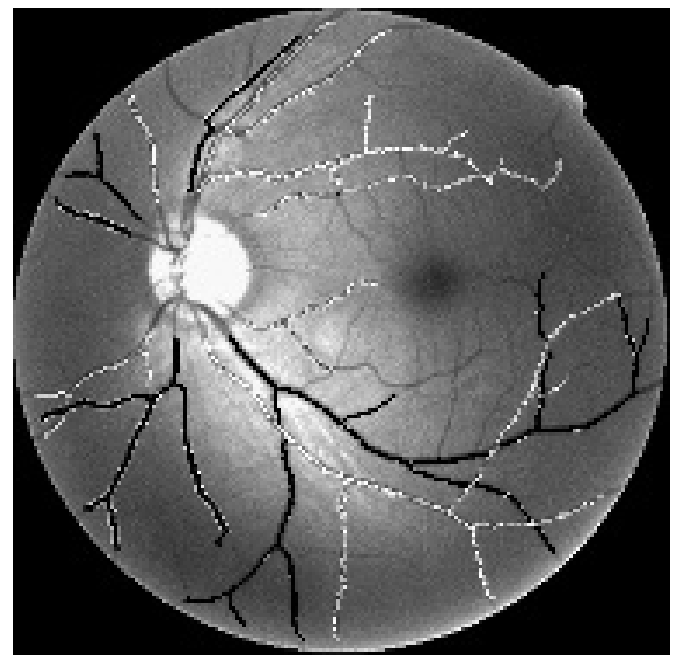

(b)

Fig. 10 (a) Detected blood vessels are shown by black lines on a black-and-white retinal image. (b) After the automatic tracking of the blood vessels from the optic disk.

too close to each other. This could lead to errors in vascular diameter measurement. Most erroneous measurements of vessel diameters occurred in the arteries; accordingly, a technique for identifying arteries and veins that is capable of reducing errors needs to be developed.

Our system detected narrowing arteriolae with a focus on an irregularity on fundus images. Moreover, it brought to light the possibility that ophthalmologists could diagnose adult diseases at a higher sensitivity by making full use of image data output from a computer.

\section{CONCLUSION}

In this study, a new scheme for an automated blood vessel analysis was presented using digitized retinal fundus images. This scheme might be applied to the computer-aided diagnosis (CAD) system for diagnosing diseases detected 
in the eyes and other organs. The results of the preliminary testing showed a desirable consistency with those obtained from the proposed scheme. It was demonstrated that the algorithm analyzed the distributions and geometries of retinal blood vessels with higher accuracy and reliability. The result of the initial work on 70 fundus images made it clear that the efficiency and accuracy of blood vessel diagnosis was considerably improved. The results of this study will be sent to ophthalmologists for further evaluation. The efficiency and accuracy of blood vessel diagnosis was improved by the recognition of vascular geometries and distributions with higher accuracy. The application of the proposed scheme to fundus images enhanced CAD system performance in detecting abnormal blood vessels having narrowing arteriolae with a focus on an irregularity in the retinal images. At present, we are making an effort to modify the scheme to minimize those problems mentioned in the discussion section. In the future, the integrated analysis scheme will be further improved, and more clinical cases will be enrolled in the study to evaluate its accuracy. The techniques employed in our system will help in improving diagnostic accuracy as well as in lessening the workload of ophthalmologists in the future.

\section{ACKNOWLEDGMENTS}

The authors thank M. Lu, Y. Tsukiyama, and T. Kunieda for their great contribution to this study. This work was supported in part by a Grant-In-Aid for Scientific Research from the Ministry of Education, Culture, Sports, Science and Technology, and Softopia Japan Collaborative Research.

\section{REFERENCES}

1. Y.A. Tolias and S.M. Panas, "A Fuzzy Vessel Tracking Algorithm for Retinal Images Based on Fuzzy Clustering”, IEEE Transactions on Medical Imaging, 17 (2), 263-273, 1998.

2. F. Zana and J.C. Klein, "A multimodal Registration Algorithm of Eye Fundus Images Using Vessels Detection and Hough Transform”, IEEE Transactions on Medical Imaging, 18 (5), 419-428, 1999.

3. S. Chaudhuri, S. Chatterjee, N. Katz, M. Nelson and M. Goldbaum, "Detection of Blood Vessels in Retinal Images Using Two-dimentional Matched Filters", IEEE Transactions on Medical Imaging, 8 (6), 263-269, 1989.

4. A. Can, H.S. James, N. Turner, H.L. Tanenbaum and B. Roysam, "Rapid Automated Tracing and Feature Extraction from Retinal Fundus Images Using Direct Exploratory Algorithm”, IEEE Transactions on Biomedicine, 3 (2), 125-138, 1999.

5. S. Sakuma, T. Nakanishi, Y. Takahashi, Y. Fujino, S. Ohtsuka, A. Tomono, N. Nakanishi, T. Tsubouchi and T. Tanino, "Image Registration, Color Correction and Change Detection Based on Value of Difference in Sequential Ocular Fundus Images", The IEICE (Institute of Electronics, Information and Communication Engineers) Transaction on Information and Systems, PT2 (Japanese Edition), J-84-D-II (10), 2247-2257, 2001.

6. K. Sugio, T. Kunieda, H. Fujita, T. Hara, T. Kawase, K. Ogawa, A. Ishida and M. Inagaki, "Analysis of Blood Vessel Intersections in Fundus Images”, Japanese Journal of Radiological Technology, 56 (3), 507-509, 2000.

7. J. Hayashi, T. Kunieda, J. Cole, R. Soga, Y. Hatanaka, M. Lu, T. Hara and H. Fujita, "A Development of Computer-aided Diagnosis System Using Fundus Images", Proc. of 7th International Conference on Virtual System and MultiMedia, Edited by H. Thwaites and L. Addison, 429-438, IEEE Computer Society, Los Alamitos, 2001.

8. K.R. Hoffmann, K. Doi, H.P. Chan, L. Fencil, H. Fujita and A. Muraki, "Automated Tracking of the Vascular Tree in DSA Images Using a Double-square-box Region-of Search Algorithm”, Proc. of SPIE-The International Society for Optical Engineering, Medicine XIV / PACS IV, 626, 326-333, 1986.

*hatanaka@gifu-nct.ac.jp; phone 8158320 1384; fax 8158320 1263; gifu-nct.ac.jp 